\title{
Acupuntura em trabalhadores: revisão integrativa da literatura
}

\section{Acupuncture in workers: an integrative literature review}

Danielli Rafaeli Candido Pedro', Beatriz Maria dos Santos Santiago Ribeiro²

1. ORCID: https://orcid.org/0000-0003-4141-1220. Doutoranda em Enfermagem pela Universidade Estadual de Londrina. Londrina, Paraná, Brasil. E-mail: danirafaeli@ hotmail.com.

2. ORCID: https://orcid.org/0000-0001-5211-5422. Doutoranda em Enfermagem pela Escola de Enfermagem de Ribeirão Preto da Universidade Estadual de São Paulo. Docente do Departamento de Enfermagem da Faculdade de Apucarana. Califórnia, Paraná, Brasil. E-mail: beatrizsantiago1994@hotmail.com.

CONTATO: Beatriz Maria dos Santos Santiago Ribeiro. | Endereço institucional: Rua Osvaldo de Oliveira, 600 - Jardim Flamingos, Apucarana, Paraná, Brasil. Telefone: (43) 99172-4895. - E-mail: beatrizsantiago1994@hotmail.com.

RESUMO Objetivou-se analisar as evidências científicas disponíveis na literatura sobre o uso da acupuntura em trabalhadores. Estudo de revisão integrativa da literatura, com busca realizada em agosto de 2019, nas bases de dados Literatura Latino-Americana e do Caribe em Ciências da Saúde, na Biblioteca Virtual em Saúde e na Biblioteca Eletrônica Científica Online. Com os descritores controlados, Saúde do trabalhador; Acupuntura e Trabalhadores. Foram incluídos artigos primários, em português, disponíveis na integra e publicados entre os anos de 2009 e 2019 e foram excluídos artigos secundários. Compuseram a amostra final três artigos, os quais abordavam o uso da acupuntura em trabalhadores de diferenciadas amostras. A acupuntura mostrou-se uma estratégia eficaz para melhoria das condições de saúde e qualidade de vida dos trabalhadores. A acupuntura pode ser uma aliada na promoção de saúde ou tratamento de doenças, há necessidade de maiores evidências cientificas.

DESCRITORES: Saúde do Trabalhador. Acupuntura. Trabalhadores. 
ABSTRACT The objective of this study was to analyze the scientific evidence available in the literature on the use of acupuncture in workers. Integrative literature review study, with a search conducted in August 2019 in the Latin American and Caribbean Literature in Health Sciences databases, the Virtual Health Library and the Online Scientific Electronic Library. With controlled descriptors, Worker health; Acupuncture and Workers. Primary articles, in Portuguese, available in full and published between 2009 and 2019, were included and secondary articles were excluded. The final sample three tree articles, which addressed the use of acupuncture in workers from different samples. Acupuncture proved to be an effective strategy for improving health conditions and quality of life for workers. Acupuncture can be an ally in health promotion or treatment of diseases, there is a need for more scientific evidence.

DESCRIPTORS: Occupational Health. Acupuncture. Workers.

\section{INTRODUÇÃO}

0 s trabalhadores podem adoecer por causas específicas relacionadas com o trabalho como os acidentes de trabalho1. Desse modo, buscar estratégias que visam atuar na promoção e proteção da saúde do trabalhador, no bem-estar e na prevenção a exposição de riscos e acidentes de trabalho, fazem necessárias pois evitam o processo de adoecimento ${ }^{2}$.

Os agravos à saúde do trabalhador não se restringem apenas a acidentes do trabalho, pois também devemos considerar as condições ambientais ou atividades de trabalho que farão com que o trabalhador adoeça ${ }^{3}$. Doenças do trabalho são o conjunto de danos ou agravos que acometem a saúde do trabalhador, sendo causados, desencadeados ou agravados por fatores de risco presente no ambiente de trabalho ${ }^{4}$.Os avanços de mercado, tecnológicos e científicos, demonstram a necessidade de maior atenção as condições legais, éticas, sociais e de saúde sobre o ambiente de trabalho e a interação do indivíduo com este meio.

O trabalho permite a formação do indivíduo, técnica, política, cultural, estética e artisticamente envolvendo a subjetividade. Em certas condições, a interação entre o trabalhador e seu trabalho pode desencadear vivências de sofrimento, entretanto proporciona vivências de prazer, pois é por meio do labor que o indivíduo constrói sua vida e se insere no mundo laboral, não somente como forma de sobrevivência, mas também para realização pessoal e profissional ${ }^{5}$.

Destaca-se que o trabalho pode afetar diretamente a saúde física, mental e social dos indivíduos. Sabe-se que o indivíduo é exposto a condições estressantes, que acarretam 
uma série de manifestações para este trabalhador e consequentemente sua qualidade de vida. Neste sentido, torna-se essencial que medidas de alívio sejam implementadas na busca pela saúde do trabalhador, já que no cenário laboral uma das alternativas incluem o uso da acupuntura por trabalhadores ${ }^{6}$.

A acupuntura é uma das técnicas da Medicina Tradicional Chinesa (MTC), que se fundamenta em uma abordagem holística da saúde, inter-relacionando o meio, aos fatores emocionais, psicossociais e biológicos, apresenta um diagnóstico energético e tratamento individualizado ${ }^{7}$. A intervenção de enfermagem deve ser desenvolvida no sentido de manter ou promover este equilíbrio, buscando-se a manutenção ou adaptação do indivíduo às mudanças ambientais ${ }^{8}$.

A acupuntura iniciou há mais de 4.000 anos e, ainda é alvo de questionamentos 9 . Sendo, uma intervenção em saúde, originada de anexo de conhecimentos teóricoempíricos da MTC, na qual aborda-se de modo integral e dinâmico o processo saúdedoença. Ainda, seu uso pode ser associado com outros recursos terapêuticos ${ }^{10}$.

Em um estudo realizado, os indivíduos entrevistados sobre os benefícios da acupuntura para a saúde referiram que essa técnica terapêutica pode ser eficaz para melhoria das enfermidades, eliminação ou diminuição de doenças, sendo uma ferramenta potencial para o desenvolvimento de ações de promoção de saúde no trabalho ${ }^{11}$.

A MTC através da acupuntura faz parte do grupo das Práticas Integrativas e Complementares (PIC), que é um conjunto de práticas de cuidado, saberes e produtos de uso terapêutico que não pertencem a medicina convencional, atuando na promoção da saúde, prevenção de agravos e de reabilitação ${ }^{12}$. A partir do ano de 2018 , através da Resolução do Conselho Federal de Enfermagem COFEN nº 585/2018, a acupuntura foi reconhecida como especialidade e/ou qualificação do profissional de enfermagem, proporcionando mais uma área de atuação deste profissional ${ }^{13}$.

A acupuntura tem por finalidade o diagnóstico de doenças e a promoção de cura a partir do estímulo da força de autocura do corpo, busca realinhar a energia por meio da estimulação de pontos corporais com agulhas, laser ou pressão. É indicada para o tratamento de múltiplas doenças, atuando beneficamente em todas as idades e níveis de atenção à saúde. Essa técnica demonstra-se eficaz para desordens físicas e de cunho psicológico e/ou emocionais ${ }^{14-15}$.

O acréscimo dessa especialização na área da enfermagem, permite refletir sobre a possibilidade de os enfermeiros serem especialistas em diversas áreas e aliarem os conhecimentos em prol do público-alvo em que esse profissional atua, por exemplo, o enfermeiro do trabalho que pode especializar-se em acupuntura e aliar ambas as práticas no ambiente laboral. 
Cabe ressaltar que há escassez de revisões atuais sobre este tema, o que torna esse trabalho relevante para identificação do atual estado da arte de produções científicas a respeito do uso de acupuntura em trabalhadores, sendo que certamente essa revisão pode servir como fonte de consulta para outros trabalhos que intentem em estudar essa temática, demonstrando sua relevância perante o cenário nacional da enfermagem. Diante do exposto este estudo objetiva analisar as evidências científicas disponíveis na literatura sobre o uso da acupuntura em trabalhadores.

\section{MÉTODO}

Trata-se de uma revisão integrativa, que seguiu as seguintes etapas: identificação do tema e estruturação da pergunta de pesquisa; definição dos critérios de inclusão; seleção dos artigos nas bases de dados científicas; avaliação e análise dos estudos selecionados; interpretação dos resultados e apresentação da revisão integrativa ${ }^{16}$.

Para estruturação da pergunta de pesquisa foi utilizada a estratégia PICO (acrônimo para population, intervention, comparison e outcomes), ou seja, população, intervenção, comparação e resutados ${ }^{17}$. A população $(P)$ foi definida como trabalhador, o segundo elemento, intervenção (I), foi acupuntura; o terceiro elemento comparação (C) não foi utilizado devido não ser objeto da questão deste estudo; e o quarto elemento resultados (O) não foi delimitado por ser o objetivo reconhecer os resultados da associação entre a saúde do trabalhador e a acupuntura.

Portanto, elaborou-se a seguinte questão norteadora: Quais as evidências científicas disponíveis sobre o uso da acupuntura em trabalhadores?

Inicialmente foi realizada consulta aos Descritores em Ciências da Saúde (DeCS), para conhecimento dos descritores universais. Deste modo, foram selecionados e utilizados os descritores controlados, em português: Saúde do trabalhador; Acupuntura e Trabalhadores, entre cada um deles foi utilizado o operador booleano "AND". A Tabela 1 demonstra de que forma a busca foi realizada nas bases de dadosLiteratura LatinoAmericana e do Caribe em Ciências da Saúde (LILACS), na Biblioteca Virtual em Saúde (BVS) e na Biblioteca Eletrônica Científica Online (Scielo). 
Tabela 1. Estratégia de busca nas bases de dados. 2019.

\begin{tabular}{lll}
\hline Local de busca & Estratégia de busca & \multicolumn{2}{c}{$\begin{array}{l}\text { Número de artigos encontrados na pri- } \\
\text { meira busca }\end{array}$} \\
\hline LILACS & $\begin{array}{l}\text { "Saúde do trabalhador" AND "Acu- } \\
\text { puntura" AND "Trabalhadores" }\end{array}$ & 06 \\
BVS & $\begin{array}{l}\text { "Saúde do trabalhador" AND "Acu- } \\
\text { puntura" AND "Trabalhadores" }\end{array}$ & 15 \\
SCIELO & $\begin{array}{l}\text { "Saúde do trabalhador" AND "Acu- } \\
\text { puntura" AND "Trabalhadores" }\end{array}$ & 02 \\
\hline
\end{tabular}

Fonte: Dados da pesquisa (2019).

Os critérios de inclusão estabelecidos foram artigos primários completos, no idioma português, disponíveis na íntegra, produzidos entre os anos de 2009 a 2019, como forma de obtenção de um panorama sobre o uso da acupuntura em trabalhadores na última década. Os critérios de exclusão foram estudos secundários de qualquer origem.

Para obtenção dos dados, utilizou-se uma planilha, composta de itens relativos à identificação do artigo, tipo de estudo, nível de evidência das pesquisas, objetivos e principais resultados.

Para esta revisão a classificação dos níveis de evidência utilizado foi: nível I (mais forte): revisão sistemática ou metanálise de ensaios clínicos randomizados controlados ou provenientes de diretrizes clínicas baseadas em revisões sistemáticas de ensaios clínicos randomizados controlados; nível II: pelo menos um ensaio clínico randomizado controlado bem delineado; nível III: ensaios clínicos bem delineados sem randomização; nível IV: estudos de coorte e de casocontrole bem delineados; nível V: revisão sistemática de estudos descritivos e qualitativos; nível VI: um único estudo descritivo ou qualitativo; nível VII: opinião de autoridades e/ou relatório de comitês de especialistas .

Os artigos foram analisados de forma descritiva e sintetizados em um quadro, com informações relevantes, sendo apresentada uma síntese de cada estudo.Como não foram realizadas pesquisas em seres humanos, nem utilizados dados confidenciais, institucionais ou pessoais, sendo que toda a pesquisa foi baseada em dados secundários de estudos publicados em bases de dados eletrônicas não foi necessária aprovação por parte do comitê de ética em pesquisa.

\section{RESULTADOS}

A coleta de dados foi realizada em duas etapas. A primeira consistiu na busca avançada nas bases de dados, sendo encontrados: LILACS, 6 artigos, BVS 15 estudos e Scielo 2 estudos, totalizando 23 estudos. Após o processo de seleção e identificação dos artigos que obedeceram aos critérios de inclusão estabelecidos, prévia leitura de todos os títulos e resumos, selecionaram-se publicações, sendo: LILACS, 2 artigos; BVS 1 artigo e Scielo 1 artigo. Na segunda etapa, procedeu-se à leitura na íntegra, sendo que não foram excluídos nenhum artigo pois todos contribuiam para a temática estudada, resultando a amostra de 4 artigos. A Figura 1 traz a representação gráfica do fluxo de seleção dos artigos. 
Figura 1. Fluxo de seleção dos artigos para compor a amostra da revisão integrativa. 2019.

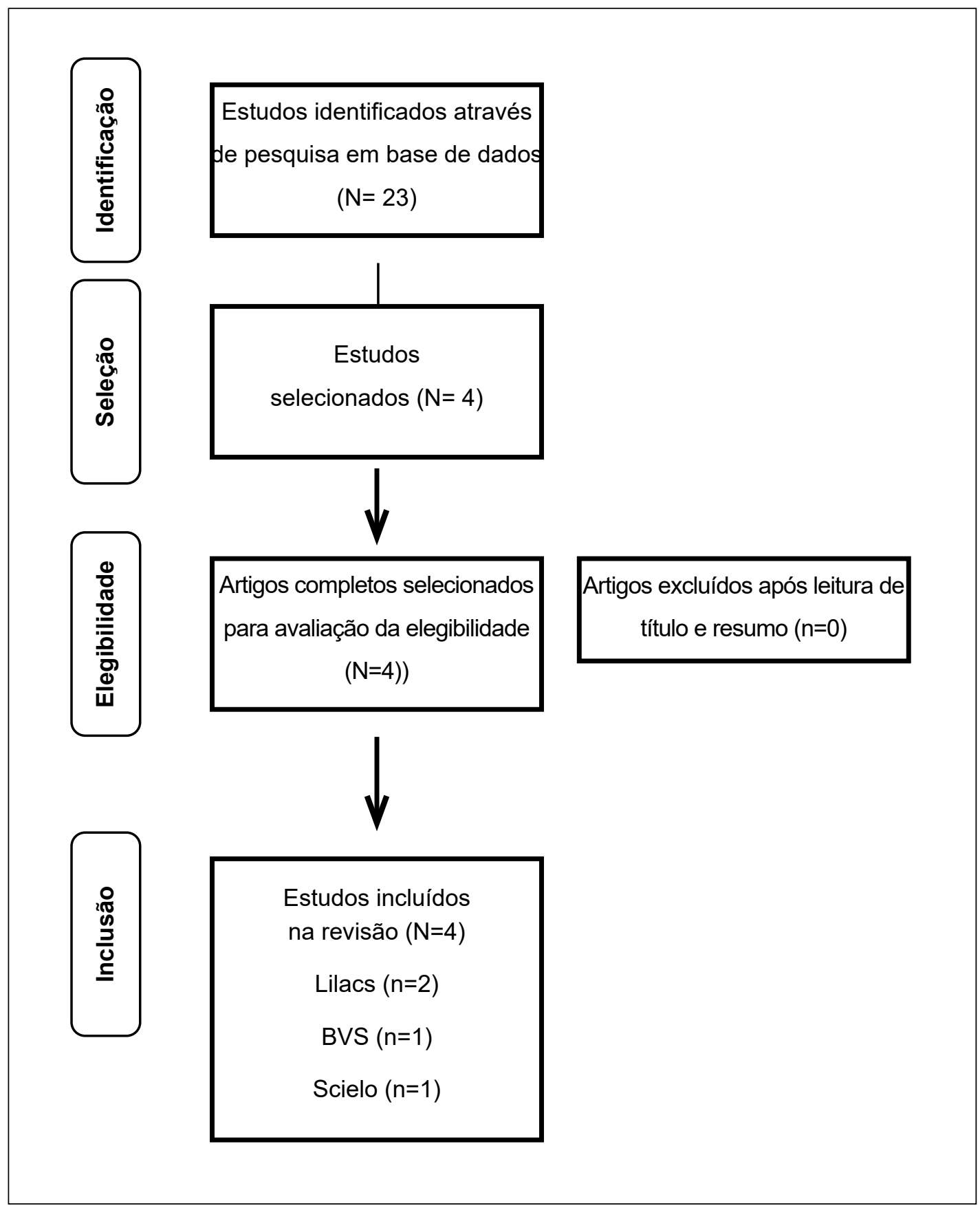

Fonte: Elaborado pelos autores (2019).

Desta maneira, a amostra final foi constituída por três artigos que se encaixavam aos critérios de inclusão. Eles estão descritos no Quadro 1, de forma a elucidar o título da produção, autores, ano de publicação, os objetivos de cada estudo e os principais resultados. 
Quadro 1. Síntese dos estudos incluídos na revisão integrativa. 2019.

\begin{tabular}{|c|c|c|c|}
\hline Título & Autor/Ano & Objetivo & Principais resultados \\
\hline $\begin{array}{c}\text { A craniopuntura } \\
\text { japonesa como } \\
\text { instrumento para o } \\
\text { tratamento da dor } \\
\text { não específica em } \\
\text { profissionais de } \\
\text { saúde. }\end{array}$ & $\begin{array}{c}\text { Barreiros, Raphael } \\
\text { Neves; Dutra, Luana } \\
\text { Borges; Silva, Ro- } \\
\text { berto Carlos Lyra da; } \\
\text { Ribeiro, Yonara Cris- } \\
\text { tiane; Moura, Lidiane } \\
\text { da Fonseca; Louro, } \\
\text { Thiago Quinellato. } \\
2019 .\end{array}$ & $\begin{array}{c}\text { Caracterizar a } \\
\text { técnica de cranio- } \\
\text { puntura japonesa } \\
\text { como instrumento } \\
\text { para o tratamento } \\
\text { da dor não espe- } \\
\text { cífica em profissio- } \\
\text { nais de saúde. }\end{array}$ & $\begin{array}{l}\text { A técnica de craniopun- } \\
\text { tura japonesa auxiliou na } \\
\text { redução da dor ( } p=0,009) \\
\text { em } 100 \% \text { dos participan- } \\
\text { tes deste estudo. }\end{array}$ \\
\hline $\begin{array}{l}\text { Qualidade de sono } \\
\text { de trabalhadores } \\
\text { obesos de um hospi- } \\
\text { tal universitário: acu- } \\
\text { puntura como terapia } \\
\text { complementar. }\end{array}$ & $\begin{array}{l}\text { Haddad, Mariana Lou- } \\
\text { renço; Medeiros, Mar- } \\
\text { celo; Marcon, Sonia } \\
\text { Silva. } 2012 .\end{array}$ & $\begin{array}{l}\text { Verificar o efeito da } \\
\text { acupuntura na qua- } \\
\text { lidade de sono de } \\
\text { trabalhadores obe- } \\
\text { sos em um hospital } \\
\text { universitário }\end{array}$ & $\begin{array}{l}\text { A acupuntura produziu um } \\
\text { efeito positivo sobre a qua- } \\
\text { lidade do sono na amostra } \\
\text { estudada. }\end{array}$ \\
\hline $\begin{array}{l}\text { Acupuntura e apeti- } \\
\text { te de trabalhadores } \\
\text { obesos de um hos- } \\
\text { pital universitário }\end{array}$ & $\begin{array}{l}\text { Haddad, Mariana } \\
\text { Lourenço and Mar- } \\
\text { con, Sonia Silva. } \\
2011 .\end{array}$ & $\begin{array}{l}\text { Mensurar o com- } \\
\text { portamento da } \\
\text { sensação de ape- } \\
\text { tite dos sujeitos } \\
\text { antes, durante e } \\
\text { após a intervenção } \\
\text { de acupuntura }\end{array}$ & $\begin{array}{l}\text { Demonstraram que o } \\
\text { comportamento do apetite } \\
\text { em relação à saciedade, } \\
\text { plenitude, desejo por ali- } \\
\text { mentos doces e palatáveis } \\
\text { sofreu modificações du- } \\
\text { rante a intervenção. Não } \\
\text { houve redução de peso } \\
\text { ou índice de massa corpó- } \\
\text { rea dos sujeitos, contudo } \\
\text { observou-se redução sig- } \\
\text { nificativa na razão cintura- } \\
\text {-quadril ( } p=0,02 \text { ) e contro- } \\
\text { le no hábito de consumir } \\
\text { alimento consolo. }\end{array}$ \\
\hline
\end{tabular}

Fonte: Elaborado pelos autores (2019).

Com relação a classificação do nível de evidência, os três estudos que compuseram a amostra foram classificados como nível VI, sendo de caráter descritivo. 


\section{DISCUSSÃO}

Os principais benefícios da acupuntura relacionados ao trabalho, são o baixo custo para aplicação, gerar efeitos colaterais mínimos, demonstrar efetividade no tratamento de dor e doenças psíquicas, sendo as mais comuns relacionadas com o trabalho, sendo assim, a técnica pode contribuir para redução de licenças, afastamentos, absenteísmo e custos com reposição de cargos ${ }^{15}$

O primeiro estudo abordado na amostra estuda e aplica uma nova técnica denominada Craniopuntura Japonesa, como instrumento para o tratamento da dor não específica em profissionais de saúde. A técnica demonstrou-se eficaz na totalidade da amostra estudada, reduzindo a dor em todos os participantes ${ }^{18}$.

As condições de trabalho, muitas vezes são precárias e levam os trabalhadores de enfermagem a exposição aos riscos ocupacionais, contribuído para o desenvolvimento de dores musculoesqueléticas. Como constatado em um estudo, que acompanhou 29 profissionais de enfermagem por 12 meses, concluindo que 96,6\% dos trabalhadores referiram dor ao longo do tempo de acompanhamento, tal dado demonstra a relevância e necessidade da implantação de técnicas de redução da dor ${ }^{19}$, como a acupuntura.

Além disso, uma revisão sistemática demonstrou que a acupuntura pode ser eficaz para alívio da dor lombar, uma das dores autor referidas por trabalhadores. Ainda relatam que muitos estudos levantados apontam para uma melhora no alívio da dor independente da técnica de acupuntura utilizada ${ }^{20}$.

Ressalta-se que um estudo descreve a implantação de um Programa de Reabilitação Ampliada, o artigo apontou a eficiência de programas de reabilitação profissional de orientação multidimensional, os serviços descritos no estudo foram: fisioterapia, terapia ocupacional, psicologia, acupuntura, reeducação postural global, tai chi chuan, massoterapia, danças circulares, grupo informativo, condicionamento físico, hidroginástica, terapias complementares e assistência social ${ }^{21}$.

A acupuntura foi empregada para trabalhadores com fibromialgia, transtornos mentais, transtornos músculo-esqueléticos e patologias mistas, mostrou-se como estratégia para minimizar o quadro álgico, sinais de fadiga, depressivos e de ansiedade e melhorar a qualidade do sono dos trabalhadores ${ }^{21}$.

Os achados corroboram com um estudo que entrevistou enfermeiras sobre o uso da acupuntura e comparou com a lista desenvolvida pela Organização Mundial de Saúde (OMS), demonstrou que a opinião das enfermeiras não difere do que é indicado, sendo que a acupuntura se mostra como uma técnica preventiva, curativa e reabilitadora, para doenças agudas ou crônicas, sendo empregada no tratamento do estresse e dores músculo-esqueléticas ${ }^{22}$. 
Outro estudo produzido no Brasil, refere que os efeitos da acupuntura sob transtornos de ansiedade têm se mostrado efetiva, podendo atuar como uma estratégia para a redução do consumo de medicamentos ${ }^{14}$.

O segundo estudo encontrado demonstrou que a acupuntura pode ser efetiva para a melhoria da qualidade de sono dos sujeitos estudados, bem como é efetiva para a promoção da qualidade de vida nos trabalhadores de enfermagem de um hospital universitário ${ }^{23}$.

Uma revisão sistemática, método aplicado em estudos com alta qualidade, demonstrou que a acupuntura tem a capacidade de oferecer excelentes resultados no tratamento de pacientes portadores de insônia, sendo amplamente indicada para o seu tratamento ${ }^{24}$.

A organização de trabalhos em turnos, principalmente em funções ininterruptas exigem o trabalho noturno, onde os profissionais acabam permanecendo por longos períodos acordados, desenvolvendo suas atividades laborais no período onde o corpo humano está acostumado a estar dormindo, essa inversão de períodos de trabalho e sono é adaptada, porém ao longo dos anos o trabalho noturno pode afetar o sono desses trabalhadores, sendo que a acupuntura revelou-se como uma opção viável para o tratamento da insônia em trabalhadores ${ }^{23}$.

O terceiro estudo que compõem esta revisão integrativa demonstrou que a acupuntura foi eficaz no controle do apetite dos trabalhadores obesos, houve também redução significante na razão cintura-quadril. Além disso a acupuntura pode ser adotada como cuidado de enfermagem com os trabalhadores, sendo que a técnica é de baixo custo, risco mínimo de contaminação ou infecção, e praticamente nenhum efeito colateral ${ }^{25}$.

A acupuntura pode ser empregada como uma estratégia que auxilia no processo de emagrecimento, o estímulo dos pontos de acupuntura e auriculares atuam como inibidor do apetite, tranquilizantes e reduzem a compulsividade para se alimentar ${ }^{26}$.

Através da acupuntura é possível obter uma sensação de bem-estar, melhora da função digestiva, controle e diminuição da compulsão alimentar e da ansiedade, além disso, atua na aceleração do metabolismo e liberação de hormônios que despertam sensação de bem-estar e felicidade ${ }^{27}$.

Os estudos incluídos na amostra foram classificados como de nível de evidência VI, o que indica a necessidade da produção de outros estudos com métodos mais criteriosos para a produção de evidências fortes que embasem e solidifiquem o conhecimento sobre acupuntura no trabalho. Como limitações dessa revisão, ainda não foram encontradas evidências fortes, decorrentes dos métodos do estudo e tamanho da amostra escolhida, sugerindo que mais pesquisas são necessárias para avaliar prospectivamente a implantação da acupuntura para trabalhadores. 
Vale ressaltar que estudos de revisões integrativas mostraram a necessidade de estudos sobre com foco na acupuntura e síndrome de burnout, cuja síndrome afeta somente trabalhadores, despertando e sugerindo evidências científicas que busquem identificar os melhores acupuntos e números de sessões eficazes para o tratamento de doenças do trabalho $28-29$.

Esta revisão demonstrou os diversos campos de aplicação da acupuntura para melhoria da qualidade de vida dos trabalhadores e também no tratamento de doenças. Além disso, foi elencado por um estudo o baixo custo da aplicação desta técnica, fato que impulsiona a implantação desta estratégia em instituições empregadoras como método de baixo custo e com amplas potencialidades para manutenção do trabalhador em atividade e redução com gastos por afastamento.

\section{CONSIDERAÇÕES FINAIS}

Conclui-se com esta revisão que apesar de poucas publicações sobre o tema na última década, a acupuntura mostrou-se como uma estratégia eficaz para a melhoria das condições de saúde e nutrição de trabalhadores, podendo ser empregada pelas instituições empregadoras como forma de intensificar as ações que priorizem a saúde e qualidade de vida dos trabalhadores.

Evidenciou-se neste estudo que a acupuntura atua nos mais diversos âmbitos de saúde e bem-estar, podendo ser aliada na promoção de saúde ou tratamento de doenças, como os trabalhadores estão diariamente expostos a riscos biológicos, físicos, químicos e ergonômicos, torna-se essencial que o serviço de saúde e segurança do trabalhador esteja atento as práticas que podem produzir qualidade de vida aos seus profissionais, deste modo esta revisão contribui na condensação de estudos que possam elucidar as potencialidades encontradas na acupuntura.

Há necessidade de intensificar a produção de estudos que demonstrem mais resultados sobre o uso da acupuntura em trabalhadores, capazes de produzir evidências fortes, visto que houve escassez de pesquisas nacionais sobre a temática, com elevado nível de evidência, bem como é necessário o desenvolvimento de estudos de intervenção que avaliem a longo prazo a saúde do trabalhador pós-intervenção da acupuntura. $O$ agrupamento de evidências na prática clínica da acupuntura, pode ser uma ferramenta de grande importância na implementação de ações e intervenções efetivas para a melhora da saúde do trabalhador em diversos âmbitos. 


\section{REFERÊNCIAS}

1. Ribeiro BMSS, Martins JT, Silva VA, Teston EF, da-Silva AC, Martins EAP.Occupational health nursing in civil construction: contributions based on Roy's adaptation theory. Rev Bras Med Trab [Internet]. 2019 [acesso em 2020 jan 15]; 17(2): 260-67. Disponível em: http://dx.doi.org/10.5327/Z1679443520190364.

2. Ribeiro BMSS, Hirai VHG, Teston EF. A redução de perícias médicas baseado na gestão de absenteísmo, rotatividade e qualidade de vida no trabalho. Rev Gestão \& Saúde [Internet]. 2018 [acesso em 2020 jan 15]; 9(3): 393-403. Disponível em: https://periodicos.unb.br/index.php/rgs/article/view/20291

3. Hirai VHG., Ribeiro BMSS, Novaes TB, Martins JT, Teston EF. Prevalência de dislipidemia em trabalhadores de uma empresa do setor papeleiro. Rev. Bras. Med. Trab [Internet]. 2019 [acesso em 2020 jan 15]; 17(1):54-60. Disponível em: : http://dx.doi.org/10.5327/Z1679443520190345.

4. Brasil. Ministério da Saúde. Portaria $n^{\circ}$ 1.823, de 23 agosto de 2012. Institui a Política Nacional de Saúde do Trabalhador e da Trabalhadora. Brasília: Ministério da Saúde, 2012. [acesso em 2020 jan 15]. Disponível em: https://bvsms.saude.gov.br/bvs/saudelegis/gm/2012/prt1823_23_08_2012.html.

5. Marques CRD., Ribeiro BMSS, Novaes TB, Martins JT, Dias HG, Dalri RCMB D, Bernardes MLG, Karino ME. Fatores de satisfações e insatisfações no trabalho de enfermeiros. Rev. enferm. UFPE on line [Internet]. 2020 [acesso em 2020 jul 25]; ;14:e244966. Disponível em: http://dx.doi.org/ 10.5205/1981-8963.2020.244966.

6. Viana LP, Tavares AAAC, Brugiolo PG, Carvalho JMB, Raposo NRB, Baraky LR. Efeito da acupuntura na qualidade de vida de trabalhadores portadores de zumbido expostos ao ruído: um estudo piloto. Rev Bras Med Trab. [Internet]. 2013 [acesso em 2020 jan 20]; 11(2):53-59. Disponível em: https://cdn.publisher.gn1. link/rbmt.org.br/pdf/v11n2a02.pdf.

7. Porporatti AL, Costa YM, Stuginski-Barbosa J, Bonjardim LR, Conti PCR. Protocolos de acupuntura para o tratamento da disfunção temporomandibular. Rev Dor [Internet]. 2015 [acesso em 2020 jan 15]; 16(1):5359. Disponível em: http://dx.doi.org/10.5935/1806-0013.20150011.

8. Silva SH, Ribeiro BMSS, Scorsolini-Comin F, Okamura CT, Martins EAP. Intervenções de profissionais de enfermagem para a assistência espiritual: uma revisão narrativa. Rev Elet Acer Saúde [Internet]. 2020 [acesso em 2020 jul 25]; (55):e3788. Disponível em: http://dx.doi.org/10.25248/reas.e3788.2020.

9. Ribeiro BMSS, Silva VAD, Boaretto JP, Freitas IRS, Dalri RCMB, Martins EAP. Reflexões sobre a biossegurança em acupuntura. Rev Bras Med Trab [Internet]. 2020 [acesso em 2020 jul 25]; 18(1):109112. Disponível em: http://dx.doi.org/10.5327/Z1679443520200456.

10. Rocha SP, Benedetto MACD, Fernandez, FHB, Gallian DMC. A trajetória da introdução e regulamentação da acupuntura no Brasil: memórias de desafios e lutas. Rev Ciênc. saúde Colet [Internet]. 2015 [acesso em 2020 jan 15]; 20(1):155-164. Disponível em: http://dx.doi.org/10.1590/1413-81232014201.18902013.

11. Cintra MER, Figueiredo R. Acupuncture and health promotion: possibilities in public health services. Rev Interface [Internet]. 2010 [acesso em 2020 jan 15]; 14(32):139-54. Disponível em: http://dx.doi. org/10.1590/S1414-32832010000100012

12. Azevedo C, Moura CC, Corrêa HP, Mata LRF, Chaves ÉCL, Chianca TCM. Práticas integrativas e complementares no âmbito da enfermagem: aspectos legais e panorama acadêmico-assistencial. Esc Anna Nery [Internet]. 2019 [acesso em 2020 jan 15]; 23(3):e20180389. Disponível em: http://dx.doi. org/10.1590/2177-9465-ean-2018-0389.

13. Conselho Federal de Enfermagem. Resolução COFEN N 585/2018. Estabelece e reconhece a acupuntura como especialidade e/ou qualificação do profissional de Enfermagem. Brasília (DF): COFEN; 2018. [acesso em 2020 jan 15]. Disponível em: http://www.cofen.gov.br/resolucao-cofen-no-585-2018_64784.html.

14. Goyatá SLT, Avelino CCV, Santos SVM, Junior DIS, Gurgel DSLM, Terra FS. Efeitos da acupuntura no tratamento da ansiedade: revisão integrativa. Rev Bras Enferm [Internet]. 2016 [acesso em 2020 jan 15]; 69(3):602-609. Disponível em: http://dx.doi.org/10.1590/0034-7167.2016690325i.

15. Teixeira RF. A acupuntura enquanto prática complementar na atenção à saúde do trabalhador. Universidade Federal do Espírito Santo. Mestrado em Enfermagem Profissional [dissertação]. 2015. [acesso em 2020 jan 15]. Disponível em: http://repositorio.ufes.br/handle/10/5278. 
16. Mendes KDS, Silveira RCCP; Galvão CM. Revisão integrativa: método de pesquisa para a incorporação de evidências na saúde e na enfermagem. Rev Texto contex enferm. [Internet]. 2008 [acesso em 2020 jan 15]; 17(4):758-764. Disponível em: http://dx.doi.org/10.1590/S0104-07072008000400018.

17. Santos CMC, Pimenta CAM, Nobre MR, Cuce. A estratégia PICO para a construção da pergunta de pesquisa e busca de evidências. Rev. Latino-Am. Enferm. [Internet]. 2007 [acesso em 2020 jan 15]; 15(3):508-511. Disponível em: http://dx.doi.org/10.1590/S0104-11692007000300023.

18. Barreiros RN, Dutra LB, Silva RCL, Ribeiro YC, Fonseca Moura L, Louro TQ. A craniopuntura japonesa como instrumento para o tratamento da dor não específica em profissionais de saúde. Rev Fund Care [Internet]. 2019 [acesso em 2020 jan 15]; 11(3):594-598. Disponível em: http://dx.doi.org/10.9789/21755361.2019.v11i3.594-598.

19. Santos EC, Andrade RD, Lopes SGR, Valga C. Prevalência de dor musculoesquelética em profissionais de enfermagem que atuam na ortopedia. Rev. dor [Internet]. 2017 [acesso em 2020 jan 15]; 18(4):298-306. Disponível em: http://dx.doi.org/10.5935/1806-0013.20170119.

20. Rosa R, Dias CP, Roncada C. Efeitos da acupuntura na redução da dor lombar. Rev Pesq Fisio [Internet]. 2016 [acesso em 2020 jan 15]; 6(2):167-178,. Disponível em: http://dx.doi.org/10.17267/2238-2704rpf.v6i2.872.

21. Bartilotti CB, Andrade PR, Varandas JM, Ferreira PCG; Cabral C. Programa de Reabilitação Ampliada (PRA): uma abordagem multidimensional do processo de reabilitação profissional. Rev Acta Fisiatr [Internet]. 2009 [acesso em 2020 jan 15]; 16(2):66-75. Disponível em: http://www.revistas.usp.br/actafisiatrica/article/ view/103169.

22. Kurebayashi LFS, Freitas GF, Oguisso T. Enfermidades tratadas e tratáveis pela acupuntura segundo percepção de enfermeiras. Rev. esc. enferm. USP [Internet]. 2009 [acesso em 2020 jan 15]; 43(4):930-936. Disponível em: http://dx.doi.org/10.1590/S0080-62342009000400027.

23. Haddad ML, Medeiros M, Marcon SS. Qualidade de sono de trabalhadores obesos de um hospital universitário: acupuntura como terapia complementar. Rev Esc Enferm USP [Internet]. 2014 [acesso em 2020 jan 15];46(1):82-88. Disponível em: http://dx.doi.org/10.1590/S0080-62342012000100011.

24. Filho RCS; Prado GF. Os efeitos da acupuntura no tratamento da insônia: revisão sistemática. Rev Neurocienc [Internet]. 2007 [acesso em 2020 jan 15]; 15(3):183-189. Disponível em: http://dx.doi. org/10.4181/RNC.2007.15.183.

25. Haddad ML, Marcon SS. Acupuntura e apetite de trabalhadores obesos de um hospital universitário. Acta Paul Enferm [Internet]. 2011 [acesso em 2020 jan 15]; 24(5):676-682. Disponível em: http://dx.doi. org/10.1590/S0103-21002011000500013.

26. Ornela RG, Oba MDV, Kinouchi FL, Sigoli MA, Scandiuzzi RJ, Soares DW, Carvalho PC. Acupuntura no tratamento da obesidade. J Health Sci Inst [Internet]. 2016 [acesso em 2020 jan 15]; 34(1):17-23. Disponível em: http://docs.bvsalud.org/biblioref/2016/09/1507/v34_n1_2016_p17a23.pdf.

27. Matos DC, Ramos ÉCS, Sakaguti ES. A acupuntura como recurso para o tratamento da obesidade em adolescentes: uma revisão bibliográfica. Faculdade de Educação, Ciência e Tecnologia [trabalho de conclusão de curso]. 2012. [acesso em 2020 jan 15]. Disponível em: http://www.firval.com.br/ ftmateria/1434810098.pdf.

28. Ribeiro BMSS, Santos CD, Martins EAP, Vedoato T, Silverio-lopes S, Dalri RCMB. Auriculoterapia no enfretamento da síndrome de burnout: Revisão Integrativa. Intern J Devel Res [Internet]. 2020 [acesso em 2020 jul 25]; 10(4): 35463-35466. Disponível em: http://dx.doi.org/10.37118/ijdr.18741.04.2020.

29. Ribeiro BMSS, Martins EAP, Vedoato T, Freitas IRS, Dalri RCMB. Acupuntura no tratamento da síndrome de burnout. Intern J Devel Res [Internet]. 2020 [acesso em 2020 jul 25];10(4):35474-35477. Disponível em: http://dx.doi.org/10.37118/ijdr.18752.04.2020. 